\title{
THE NONCHARACTERISTIC CAUCHY PROBLEM FOR PARABOLIC EQUATIONS IN TWO SPACE VARIABLES ${ }^{1}$
}

\author{
DAVID COLTON
}

\begin{abstract}
An integral representation is obtained for the solution of the noncharacteristic Cauchy problem for second order parabolic equations in two space variables with entire, time independent coefficients. This is accomplished through the use of contour integration techniques and the calculus of residues in the space of several complex variables.
\end{abstract}

I. Introduction. In this note we will give an integral representation of the solution to the noncharacteristic Cauchy problem for second order parabolic equations in two space variables with entire, time independent coefficients. Although improperly posed in the real domain, such problems nevertheless arise when inverse methods are used to study free boundary problems for parabolic equations, for example in the case of the inverse Stefan problem for the heat equation (cf. [2]). The noncharacteristic Cauchy problem for parabolic equations becomes properly posed if the behavior of the solution in the complex domain is taken into consideration, and hence we make use of contour integration techniques and the calculus of residues in order to obtain our desired integral representation. The representation of the solution obtained in this paper is valid in the large (in the space of several complex variables) as opposed to the local solution obtained via the Cauchy-Kowalewski theorem. This is of crucial importance as far as the applications are concerned. Such a representation will also allow us to obtain results on the analytic continuation of solutions to parabolic equations.

The problem considered here has previously been studied by C. D. Hill in [3] through the use of a one-parameter family of conformal mappings (thus making the coefficients of the differential equation dependent on time) and the construction of a new fundamental solution for parabolic equations. In Hill's work this new fundamental solution is constructed by

Received by the editors July 20, 1972 and, in revised form, November 10, 1972.

AMS (MOS) subject classifications (1970). Primary 35K15, 35R25; Secondary 35C15, 35R30.

Key words and phrases. Integral representation, Cauchy problem, parabolic equation, characteristics, complex variables.

${ }^{1}$ This research was supported in part by NSF Grant GP-27232. 
recursively solving an infinite family of complex Goursat problems for nonhomogeneous parabolic equations in two space variables. Our approach is much simpler. In particular we avoid the use of conformal mappings and construct our fundamental solution in one step through the use of a generalized version of the Cauchy-Kowalewski theorem.

In the analysis which follows $C^{2}$ denotes the space of two complex variables.

II. The noncharacteristic Cauchy problem for parabolic equations. We consider the general linear, second order parabolic equation in two space variables (with time independent coefficients) written in normal form

$$
u_{x x}+u_{y y}+a(x, y) u_{x}+b(x, y) u_{y}+c(x, y) u-d(x, y) u_{t}=0
$$

and make the assumption that the coefficients in equation (2.1) are entire functions of their independent (complex) variables. Now let $u(x, y, t)$ be an analytic solution of equation (2.1) and make the nonsingular change of variables in $C^{2}$ defined by

$$
z=x+i y, \quad z^{*}=x-i y .
$$

Note that $z^{*}=\bar{z}$ if and only if $x$ and $y$ are real. Under such a transformation equation (2.1) assumes the form

$$
\begin{aligned}
L[U]= & U_{z z^{*}}+A\left(z, z^{*}\right) U_{z}+B\left(z, z^{*}\right) U_{z^{*}} \\
& +C\left(z, z^{*}\right) U-D\left(z, z^{*}\right) U_{t}=0
\end{aligned}
$$

where

$$
\begin{aligned}
U\left(z, z^{*}, t\right) & =u\left(\frac{z+z^{*}}{2}, \frac{z-z^{*}}{2 i}, t\right), \\
A\left(z, z^{*}\right) & =\frac{1}{4}\left[a\left(\frac{z+z^{*}}{2}, \frac{z-z^{*}}{2 i}\right)+i b\left(\frac{z+z^{*}}{2}, \frac{z-z^{*}}{2 i}\right)\right], \\
B\left(z, z^{*}\right) & =\frac{1}{4}\left[a\left(\frac{z+z^{*}}{2}, \frac{z-z^{*}}{2 i}\right)-i b\left(\frac{z+z^{*}}{2}, \frac{z-z^{*}}{2 i}\right)\right], \\
C\left(z, z^{*}\right) & =\frac{1}{4} c\left(\frac{z+z^{*}}{2}, \frac{z-z^{*}}{2 i}\right), \\
D\left(z, z^{*}\right) & =\frac{1}{4} d\left(\frac{z+z^{*}}{2}, \frac{z-z^{*}}{2 i}\right) .
\end{aligned}
$$

We now introduce the adjoint equation

$$
M[V]=V_{z z^{*}}-\frac{\partial(A V)}{\partial z}-\frac{\partial(B V)}{\partial z^{*}}+C V+D V_{t}=0 .
$$


Let $V$ be a solution of $M[V]=0$ ( $V$ will be prescribed more precisely in a few moments) and use Stokes theorem to integrate the identity

$$
\begin{aligned}
V L[U]-U M[V]= & \left(A V U+\frac{1}{2} V U_{z^{*}}-\frac{1}{2} V_{z^{*}} U\right)_{z} \\
& +\left(B V U+\frac{1}{2} V U_{z}-\frac{1}{2} V_{z} U\right)_{z^{*}}-(D V U)_{t}
\end{aligned}
$$

over the torus $G \times \Omega$, where $\Omega$ is the circle $|t-\tau|=\delta>0$ in the complex $t$ plane and $G$ is a two-dimensional cell in $\left(z, z^{*}\right)$ space bounded by a noncharacteristic analytic curve $C_{3}$ and line segments $C_{1}$ and $C_{2}$ which lie in the characteristic planes $z=\zeta$ and $z^{*}=\bar{\zeta}$ and join the point $R=(\zeta, \bar{\zeta})$ to the curve $C_{3}$ at the points $Q$ and $P$ respectively (see Figure 1 below).

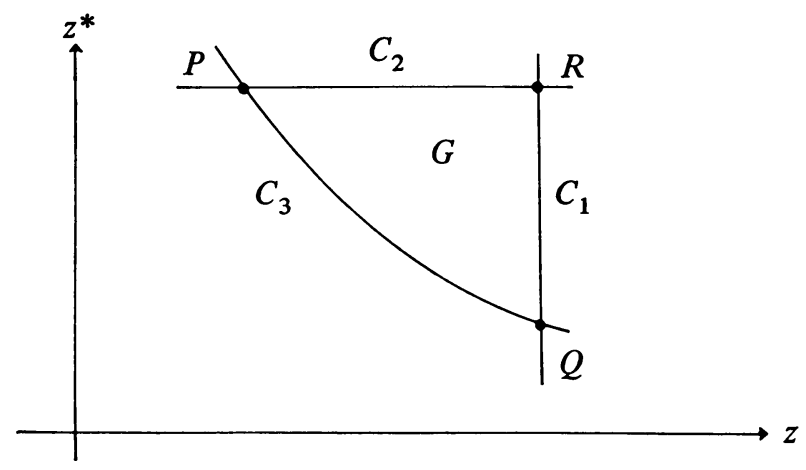

Figure 1

The result of this integration (after performing an integration by parts on the right-hand side of the resulting identity) is, in the notation of the calculus of exterior differential forms,

$$
\begin{aligned}
0= & \iint_{C_{1} \times \Omega}\left(A V-V_{z^{*}}\right) U d z^{*} d t-\iint_{C_{2} \times \Omega}\left(B V-V_{z}\right) U d z d t \\
& +\int_{\Omega}\left[V(R, t) U(R, t)-\frac{1}{2} V(P, t) U(P, t)-\frac{1}{2} V(Q, t) U(Q, t)\right] d t \\
& +\iint_{C_{3} \times \Omega}\left[\left(A V U+\frac{1}{2} V U_{z^{*}}-\frac{1}{2} V_{z^{*}} U\right) d z^{*} d t\right. \\
& \left.-\left(B V U+\frac{1}{2} V U_{z}-\frac{1}{2} V_{z} U\right) d z d t\right],
\end{aligned}
$$

where we have made use of the fact that $d z d z^{*}=0$ on $\partial C \times \Omega$. Note that an expression of the form $U(R, t)$ is a function of three independent variables, i.e. $U(R, t)=U(\zeta, \bar{\zeta}, t)$ where $(\zeta, \bar{\zeta})$ are the Cartesian coordinates of the point $R$ in $C^{2}$. 
We now want to choose $V$ and the domain $G$ such that equation (2.7) reduces to an expression for the solution of equation (2.1) satisfying prescribed Cauchy data on a noncharacteristic analytic surface $S$.

We first assume that the intersection of the plane $t=\tau$ with the surface $S$ is a one-dimensional curve $C_{3}^{\prime}=C_{3}^{\prime}(\tau)$. Suppose $C_{3}^{\prime}$ is described by the equation $F(x, y ; \tau)=0$. Since $S$ is analytic we can write

$$
F\left(\frac{z+z^{*}}{2}, \frac{z-z^{*}}{2 i} ; \tau\right)=0
$$

and this is the equation for $C_{3}^{\prime}$ in $\left(z, z^{*}\right)$ space. We will choose $C_{3}=C_{3}(\tau)$ to be an analytic curve lying on this complex extension of $C_{3}^{\prime}(\tau)$ and intersecting the characteristic planes $z=\zeta$ and $z^{*}=\bar{\zeta}$ at the points $Q$ and $P$, respectively. We now turn our attention to the construction of $V$. In particular we ask that $V=V\left(z, z^{*} ; \zeta, \bar{\zeta} ; t-\tau\right)$ be an entire function of $z, z^{*}$ and $t-\tau$, except for a simple pole with residue one at the point $\left(z, z^{*}, t\right)=(\zeta, \bar{\zeta}, \tau)$. We also ask that $V$ satisfy the initial conditions

$$
V_{z^{*}}=A V
$$

on $C_{1} \times \Omega$, and

$$
V_{z}=B V
$$

on $C_{2} \times \Omega$. The function $V$ can be constructed in the following manner. In equation (2.5) let $t-\tau=1 / \xi$. Then equation (2.5) becomes

$$
W_{z z^{*}}-\partial(A W) / \partial z-\partial(B W) / \partial z^{*}+C W-\xi^{2} D W_{\xi}=0
$$

where $W\left(z, z^{*} ; \zeta, \bar{\zeta} ; \xi\right)=V\left(z, z^{*} ; \zeta, \bar{\zeta} ; 1 / \xi\right)$. Integrating equations (2.8) and (2.9) along the characteristics, and requiring $V$ to have a simple pole with residue one at $\left(z, z^{*}, t\right)=(\zeta, \bar{\zeta}, \tau)$, gives

$$
\begin{gathered}
V\left(\zeta, z^{*} ; \zeta, \bar{\zeta} ; t-\tau\right)=\frac{1}{t-\tau} \exp \left\{\int_{\zeta}^{z^{*}} A(\zeta, \sigma) d \sigma\right\}, \\
V(z, \bar{\zeta} ; \zeta, \bar{\zeta} ; t-\tau)=\frac{1}{t-\tau} \exp \left\{\int_{\zeta}^{z} B(\sigma, \bar{\zeta}) d \sigma\right\},
\end{gathered}
$$

From Hörmander's generalized Cauchy-Kowalewski theorem [4] we can locally construct (by iteration) a unique solution $W$ satisfying equations 
(2.10), (2.13), and (2.14). From [5] we can conclude that $W$ is in fact an entire function of $z, z^{*}$, and $\xi$. Setting $\xi=1 /(t-\tau)$ in the expression obtained in this manner for $W$ gives us the function $V\left(z, z^{*} ; \zeta, \bar{\zeta} ; t-\tau\right)$ with the desired properties.

If in equation (2.7) we now let $C_{3}=C_{3}(\tau)$ lie on the complex extension of the intersection of $S$ with the plane $t=\tau$ and let $V=V\left(z, z^{*} ; \zeta, \bar{\zeta} ; t-\tau\right)$ be the function just constructed, we have

$$
U(\zeta, \bar{\zeta}, \tau)=\frac{1}{4 \pi i} \int_{\Omega}[V(P, t) U(P, t)+V(Q, t) U(Q, t)] d t
$$

$$
\begin{array}{r}
-\frac{1}{2 \pi i} \iint_{C 3(r) \times \Omega}\left[\left(A V U+\frac{1}{2} V U_{z^{*}}-\frac{1}{2} V_{z} *^{*}\right) d z^{*} d t\right. \\
\left.-\left(B V U+\frac{1}{2} V U_{z}-\frac{1}{2} V_{z} U\right) d z d t\right] .
\end{array}
$$

For $(\zeta, \bar{\zeta})$ sufficiently near the initial surface $S$, and for $\delta$ sufficiently small, equation (2.15) gives the desired integral representation of the solution to the noncharacteristic Cauchy problem for equation (2.1), provided we first deform the surface $C_{3} \times \Omega$ until it lies on the complex extension of the initial surface $S$. (Recall from equation (2.4) that $U(z, \bar{z}, t)=u(x, y, t)$.) Equation (2.15) can now be used to obtain a global solution via the straightforward use of analytic continuation of the Cauchy data and deformation of the region of integration. In particular such a procedure yields results on the analytic continuation of solutions to parabolic equations along characteristic hyperplanes in terms of the domain of regularity of the Cauchy data of these solutions along noncharacteristic analytic surfaces. For example if for each fixed $t$ in a (complex) neighborhood of $t=\tau$ the Cauchy data for $U\left(z, z^{*}, t\right)$ is regular in a domain $D$ which is conformally symmetric (cf. [1]) with respect to $C_{3}^{\prime}(\tau)$, then we can conclude that the restriction of $U\left(z, z^{*}, t\right)$ to the plane $t=\tau$ is an analytic function of $z$ and $z^{*}$ in $D \times D^{*}$ where $D^{*}=\left\{z^{*}: z^{*} \in D\right\}$. It is of interest to contrast this result with the corresponding theorem for elliptic equations obtained in [1].

\section{REFERENCES}

1. D. Colton, Cauchy's problem and the analytic continuation of solutions to elliptic equations, Proc. Sympos. Non-Well Posed Problems and Logarithmic Convexity, Springer-Verlag Lecture Note Series, vol. 316, 1973, pp. 55-66.

2. C. D. Hill, Parabolic equations in one space variable and the non-characteristic Cauchy problem, Comm. Pure Appl. Math. 20 (1967), 619-633. MR 35 \#5774.

3. - A method for the construction of reflection laws for a parabolic equation, Trans. Amer. Math. Soc. 133 (1968), 357-372. MR 38 \#3597. 
4. L. Hörmander, Linear partial differential operators, Die Grundlehren der math. Wissenschaften, Band 116, Academic Press, New York; Springer-Verlag, Berlin, 1963. MR 28 \#4221.

5. J. Persson, Linear Goursat problems for entire functions when the coefficients are variable, Ann. Scuola Norm. Sup. Pisa (3) 23 (1969), 87-98. MR 42 \#8055.

Department of Mathematics, Indiana University, Bloomington, Indiana 47401 